\title{
PET Imaging for Endocrine Malignancies: From Woe to Go
}

\author{
David Taïeb ${ }^{1}$, Rodney J. Hicks² , and Karel Pacak ${ }^{3}$ \\ ${ }^{I}$ Department of Nuclear Medicine, La Timone University Hospital, CERIMED, Aix-Marseille Univ, Marseille, France; ${ }^{2}$ Cancer \\ Imaging and Neuroendocrine Tumour Service, Peter MacCallum Cancer Centre, and Sir Peter MacCallum Department of Oncology, \\ University of Melbourne, Melbourne, Australia; and ${ }^{3}$ Section on Medical Neuroendocrinology, Eunice Kennedy Shriver National \\ Institute of Child Health and Human Development, National Institutes of Health, Bethesda, Maryland
}

$\mathbf{O}$ ver the past decade, there have been significant advances in endocrine tumor imaging using various radiopharmaceuticals. For many years, planar imaging and SPECT with ${ }^{131} \mathrm{I}$ and ${ }^{123} \mathrm{I},{ }^{99 \mathrm{~m}} \mathrm{Tc}$-sestamibi, ${ }^{111} \mathrm{In}$-pentetreotide, and ${ }^{123 / 131} \mathrm{I}$-metaiodobenzylguanidine were the only scintigraphic techniques available for imaging specific tumor types, but their diagnostic performance was inconsistent across the myriad of endocrine-related cancers. Recently, new PET agents have been introduced into clinical practice to enhance the sensitivity and specificity of nuclear imaging of these conditions. These agents provide an endocrine tumor-specific characterization that now also includes characterization of specific metabolic pathways that might be targeted for their treatment. Information provided by PET/CT is widely dependent on tumor type.

Endocrine tumors are broadly categorized into 3 groups (Fig. 1). Group A includes those that are associated mainly with an exacerbation of endocrine gland-specific hormone production signaling, generally have low proliferative signaling, and are consequently almost always benign (pituitary, parathyroid, thyroid, and adrenocortical adenomas). Group B includes those that are associated with moderate to high and sustained proliferative signaling, can either be secreting or nonsecreting, and range from indolent to aggressive behaviors (adrenocortical cancers, thyroid cancer of follicular origin, medullary thyroid carcinoma, gastroenteropancreatic neuroendocrine tumors [NETs]). Group $\mathrm{C}$ includes those that are associated mainly with constitutive impairment of cellular metabolism, are generally benign, but can undergo malignant transformation (oncocytoma, pheochromocytoma, paraganglioma).

Current PET/CT radiopharmaceuticals can be categorized into 4 main classes (Fig. 1): substrates that reflect metabolic reprogramming to compensate for increased growth requirements or inefficient substrate use $\left({ }^{18} \mathrm{~F}-\mathrm{FDG},{ }^{11} \mathrm{C}\right.$-methionine, ${ }^{18} \mathrm{~F}$-fluorocholine); precursors for cellular products, especially hormone and bioamine production $\left({ }^{18} \mathrm{~F}-\mathrm{DOPA},{ }^{11} \mathrm{C}\right.$-metomidate, $\left.{ }^{124} \mathrm{I}\right)$; receptor ligands $\left({ }^{68} \mathrm{Ga}-\right.$ and ${ }^{64} \mathrm{Cu}$-somatostatin analogs [SSAs], ${ }^{68} \mathrm{Ga}$-labeled glucagonlike peptide-1 receptor analogs, $\mathrm{C}-\mathrm{X}$-C chemokine receptor type 4-targeting radiopharmaceuticals); and antigenic recognition radiopharmaceuticals (radiolabeled monoclonal antibodies and antibody fragments). Some radiopharmaceuticals can serve as theranostic agents for internal targeted radiotherapy.

Received Jan. 8, 2017; revision accepted Feb. 14, 2017.

For correspondence or reprints contact: David Taïeb, Department of Nuclear Medicine, La Timone University Hospital, European Center for Research in Medical Imaging, Aix-Marseille University, 264 rue Saint-Pierre, 13385 Marseille, France.

E-mail: david.taieb@ap-hm.fr

Published online Feb. 23, 2017.

COPYRIGHT @ 2017 by the Society of Nuclear Medicine and Molecular Imaging. DOI: 10.2967/jnumed.117.189688
Despite the growing clinical relevance of ${ }^{18} \mathrm{~F}$-FDG PET/CT in the evaluation of endocrine tumors, little is known about the molecular determinants of tracer uptake across different endocrine tumor subtypes. However, certain pheochromocytomas, paragangliomas, and oncocytomas are among the most ${ }^{18} \mathrm{~F}$-FDG-avid tumors, irrespective of their malignancy. Pheochromocytomas and paragangliomas, especially those with an underlying mutation coding for the mitochondrial complex II (succinate dehydrogenase complex, collectively named SDH), usually exhibit highly elevated uptake on ${ }^{18} \mathrm{~F}-\mathrm{FDG}$ PET/CT. SDH deficiency leads to a truncated tricarboxylic acid cycle with accumulation of succinate, which through inhibition of prolyl hydroxylase activity stabilizes hypoxia-inducible factors that upregulate hypoxiarelated target genes, including those involved in glycolytic metabolism (1). This process leads to augmented ${ }^{18} \mathrm{~F}$-FDG uptake. SDH mutations also lead to loss of certain differentiated features, such as ${ }^{18} \mathrm{~F}-\mathrm{DOPA}$ uptake (2). Oncocytomas or tumors that contain an important oncocytic cell component also exhibit marked ${ }^{18} \mathrm{~F}-\mathrm{FDG}$ uptake. These cells are characterized by the accumulation of respiration-defective mitochondria, with increased biogenesis as a compensatory mechanism to prevent metabolic crisis. In group A, ${ }^{18} \mathrm{~F}-\mathrm{FDG}$ uptake is absent or usually very low, with the exception of some adrenocortical adenomas and pituitary adenomas. In thyroid cancer, medullary thyroid cancer, and gastroenteropancreatic NETs, ${ }^{18} \mathrm{~F}-\mathrm{FDG}$ uptake is dependent mainly on tumor aggressiveness and can therefore be used as a prognostic factor in patients with metastases (3). This metabolic pattern perhaps illustrates the need for tumor growth to increase cellular biomass and energy (e.g., adenosine triphosphate) production via increased carbohydrate metabolism. In contrast, in adrenocortical carcinoma, there has been no correlation between ${ }^{18}$ F-FDG uptake at diagnosis, Weiss score, and patient survival.

Throughout the past $5 \mathrm{y}$, new radiopharmaceuticals have been introduced in the evaluation of group A tumors. ${ }^{11} \mathrm{C}$-methionine has shown excellent results for detecting pituitary and parathyroid adenomas. Additionally, parathyroid adenomas were also found to concentrate ${ }^{18} \mathrm{~F}$-fluorocholine (4). These different modalities have established a function in the localization of these tumors and the application of targeted surgical approaches (5). Adrenocortical adenomas and some adrenocortical carcinomas can also be imaged by radiolabeled metomidate, which targets the adrenocortical enzymes CYP11B1 and CYP11B2. This approach is increasingly recognized for its ability to localize aldosterone-producing adrenocortical adenoma, which can be millimetric in size (6). Although ${ }^{11} \mathrm{C}$-metomidate has been the main agent in this regard, an iodinated ligand has also been described, opening the way for theranostic application (7). 


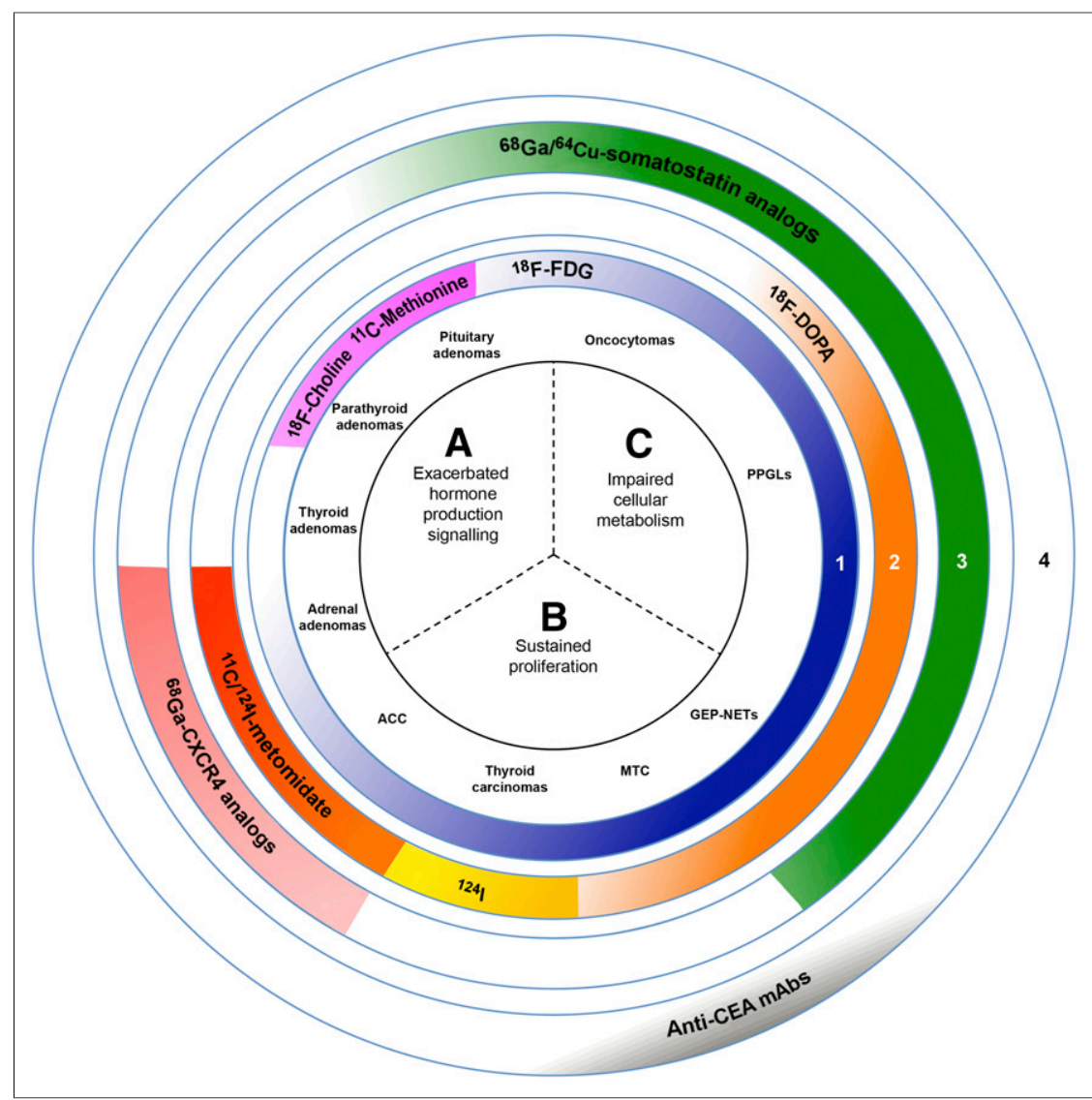

FIGURE 1. PET radiopharmaceuticals for endocrine tumor imaging and theranostics. $A-C$ are the 3 main groups of endocrine tumors, and $1-4$ are the 4 main classes of PET/CT radiopharmaceuticals.

${ }^{124}$ I-iodide PET/CT was also found to be superior to post-therapy ${ }^{131}$ I whole-body scanning and neck SPECT/CT in the detection of thyroid carcinoma metastasis (8). However, it is still unclear whether the detection of numerous other foci has any prognostic value. Nevertheless, ${ }^{124}$ I PET/CT can be proposed for patients before salvage surgery, or when 3-dimensional dosimetry is required for personalized radioiodine therapy. Finally, ${ }^{18} \mathrm{~F}$-FDOPA $\mathrm{PET} / \mathrm{CT}$ has been used extensively in the evaluation of different neuroendocrine malignancies. Although ${ }^{18} \mathrm{~F}$-FDOPA PET/CT is a highly sensitive imaging method for NETs, imaging patterns vary between tumors with different profiles. A high-uptake, prolongedretention pattern is usually observed with tumors secreting catecholamine (pheochromocytoma and paraganglioma) and serotonin (midgut NETs), possibly because of the combination of elevated expression of L-type amino acid transporters and aromatic L-amino acid decarboxylase. A moderate- to low-uptake, short-retention pattern is observed in medullary thyroid cancer and in lung and pancreatic NETs (9).

The third class of radiopharmaceuticals serves not only as diagnostic agents but also as theranostic agents mirroring ex vivo histologic classification on a whole-body, in vivo, scale. The term theranostics encapsulates the integration of diagnostic and therapeutic functions within the same platform of pharmaceuticals (theranostic pair). Implicit in the theranostic paradigm is the assumption that results derived from an imaging study using a compound labeled with a diagnostic radionuclide can precisely determine whether an individual patient is likely to benefit from a specific treatment using the same or a related compound labeled with a therapeutic radionuclide. ${ }^{68} \mathrm{Ga}$-DOTA-SSA was found to be more sensitive than ${ }^{18} \mathrm{~F}$ FDOPA in pheochromocytoma and paraganglioma regardless of its genotype $(10,11)$, and in pancreatic NETs, and both radiopharmaceuticals are very sensitive in midgut NETs $\left({ }^{68} \mathrm{Ga}\right.$-DOTA-SSA being similar or slightly inferior to ${ }^{18}$ F-FDOPA) (David Taïeb, unpublished data, 2015). The use of ${ }^{68} \mathrm{Ga}$-DOTASSA is currently recommended for imaging of all well-differentiated NETs in adults and children and for selecting candidates for peptide receptor radionuclide therapy with ${ }^{177} \mathrm{Lu}$ or ${ }^{90}$ Y-DOTA-SSA $(12,13)$. Recent Food and Drug Administration approval of diagnostic use of a ${ }^{68} \mathrm{Ga}$-DOTA-SSA, and the publication of the NETTER-1 trial (14), demonstrating the efficacy of ${ }^{177}$ Lu-DOTA-SSA are likely to make this theranostic approach to NETs more widely available. More recently, other approaches have been developed for endocrine tumor imaging using somatostatin antagonists, such as glucagonlike peptide-1 radiotracers, $\mathrm{C}-\mathrm{X}-\mathrm{C}$ chemokine receptor type 4-targeting radiopharmaceuticals, and immuno-PET. Recent studies have shown that radiolabeled somatostatin receptor antagonists are preferable to agonists for in vivo peptide receptor targeting, and there are several ongoing studies across various NETs. Glucagon-like peptide-1 receptor imaging using ${ }^{68} \mathrm{Ga}$-exendin-4 PET/CT (15) and ${ }^{18} \mathrm{~F}$-radiolabeled analogs of exendin- 4 PET/CT has emerged and is highly sensitive for detecting benign insulinomas that might be missed by other imaging studies (16). Studies have also shown a high expression of C-X-C chemokine receptor type 4 in normal adrenal cortex and adrenocortical tumors, opening new avenues for imaging these tumors with peptides targeting C-X-C chemokine receptor type $4\left({ }^{68} \mathrm{Ga}\right.$-pentixafor) and theranostic approaches $(17,18)$. Finally, immuno-PET against carcinoembryonic antigen has also been studied in the evaluation of medullary thyroid cancer, with promising results (19).

These examples demonstrate that beyond a pure localization value, nuclear imaging provides unique opportunities for better characterization of endocrine tumors at the molecular level. This unique characteristic of nuclear imaging has recently been augmented by several excellent PET radiopharmaceuticals. These agents target different functional and molecular pathways, which often reflect the diverse molecular genetic landscape of endocrine tumors. Classifications of tumors have historically been based on clinical signs as correlated with autopsy, pathologic, and physiologic findings (an anatomicclinical approach). More recently, endocrine tumor-specific genetics, epigenetics, and imaging have been integrated into the comprehensive characterization of these tumors, resulting in better understanding of them and better evaluation, treatment, and follow-up. Thus, imaging phenotypes of endocrine tumors might serve as a unique template for integrating nuclear imaging into a molecular approach to classifying not only endocrine tumors but malignancies of other origins as well. This 
approach should be evaluated and expanded in clinical and research settings that aim to reclassify diseases.

\section{DISCLOSURE}

No potential conflict of interest relevant to this article was reported.

\section{REFERENCES}

1. Sullivan LB, Gui DY, Vander Heiden MG. Altered metabolite levels in cancer: implications for tumour biology and cancer therapy. Nat Rev Cancer. 2016;16: $680-693$.

2. Timmers HJ, Chen CC, Carrasquillo JA, et al. Comparison of ${ }^{18}$ F-fluoro-L-DOPA, ${ }^{18} \mathrm{~F}$-fluoro-deoxyglucose, and ${ }^{18} \mathrm{~F}$-fluorodopamine PET and ${ }^{123} \mathrm{I}-\mathrm{MIBG}$ scintigraphy in the localization of pheochromocytoma and paraganglioma. J Clin Endocrinol Metab. 2009;94:4757-4767.

3. Binderup T, Knigge U, Loft A, Federspiel B, Kjaer A. ${ }^{18}$ F-fluorodeoxyglucose positron emission tomography predicts survival of patients with neuroendocrine tumors. Clin Cancer Res. 2010;16:978-985.

4. Lezaic L, Rep S, Sever MJ, Kocjan T, Hocevar M, Fettich J. ${ }^{18}$ F-fluorocholine PET/ CT for localization of hyperfunctioning parathyroid tissue in primary hyperparathyroidism: a pilot study. Eur J Nucl Med Mol Imaging. 2014;41:2083-2089.

5. Koulouri O, Steuwe A, Gillett D, et al. A role for ${ }^{11} \mathrm{C}$-methionine PET imaging in ACTH-dependent Cushing's syndrome. Eur J Endocrinol. 2015;173:M107-M120.

6. Mendichovszky IA, Powlson AS, Manavaki R, et al. Targeted molecular imaging in adrenal disease: an emerging role for metomidate PET-CT. Diagnostics (Basel). 2016;6(4).

7. Kvaternik H, Wanek T, Hammerschmidt F, Zolle I, Aigner R, Kuntner C. Radiosynthesis of $\left[{ }^{124} \mathrm{I}\right]$ iodometomidate and biological evaluation using small-animal PET. Mol Imaging Biol. 2014;16:317-321.

8. Ruhlmann M, Jentzen W, Ruhlmann V, et al. High level of agreement between pretherapeutic ${ }^{124} \mathrm{I}$ PET and intratherapeutic ${ }^{131} \mathrm{I}$ imaging in detecting iodinepositive thyroid cancer metastases. J Nucl Med. 2016;57:1339-1342.
9. Taïeb D, Imperiale A, Pacak K. ${ }^{18}$ F-DOPA: the versatile radiopharmaceutical. Eur J Nucl Med Mol Imaging. 2016;43:1187-1189.

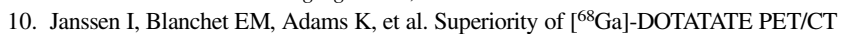
to other functional imaging modalities in the localization of SDHB-associated metastatic pheochromocytoma and paraganglioma. Clin Cancer Res. 2015;21: 3888-3895.

11. Janssen I, Chen CC, Millo CM, et al. PET/CT comparing ${ }^{68} \mathrm{Ga}$-DOTATATE and other radiopharmaceuticals and in comparison with CT/MRI for the localization of sporadic metastatic pheochromocytoma and paraganglioma. Eur J Nucl Med Mol Imaging. 2016;43:1784-1791.

12. Geijer H, Breimer LH. Somatostatin receptor PET/CT in neuroendocrine tumours: update on systematic review and meta-analysis. Eur J Nucl Med Mol Imaging. 2013;40:1770-1780.

13. Deppen SA, Liu E, Blume JD, et al. Safety and efficacy of ${ }^{68} \mathrm{Ga}$-DOTATATE PET/CT for diagnosis, staging, and treatment management of neuroendocrine tumors. J Nucl Med. 2016;57:708-714.

14. Strosberg J, El-Haddad G, Wolin E, et al. Phase 3 trial of ${ }^{177} \mathrm{Lu}$-dotatate for midgut neuroendocrine tumors. N Engl J Med. 2017;376:125-135.

15. Selvaraju RK, Velikyan I, Johansson L, et al. In vivo imaging of the glucagonlike peptide 1 receptor in the pancreas with ${ }^{68} \mathrm{Ga}$-labeled DO3A-exendin-4. $\mathrm{J}$ Nucl Med. 2013;54:1458-1463.

16. Luo Y, Pan Q, Yao S, et al. Glucagon-like peptide-1 receptor PET/CT with ${ }^{68} \mathrm{Ga}$ NOTA-exendin-4 for detecting localized insulinoma: a prospective cohort study. J Nucl Med. 2016;57:715-720.

17. Bluemel C, Hahner S, Heinze B, et al. Investigating the chemokine receptor 4 as potential theranostic target in adrenocortical cancer patients. Clin Nucl Med. 2017;42:e29-e34.

18. Herrmann K, Schottelius M, Lapa C, et al. First-in-human experience of CXCR4-directed endoradiotherapy with ${ }^{177} \mathrm{Lu}$ - and ${ }^{90} \mathrm{Y}$-labeled pentixather in advanced-stage multiple myeloma with extensive intra- and extramedullary disease. J Nucl Med. 2016;57:248-251.

19. Bodet-Milin C, Faivre-Chauvet A, Carlier T, et al. Immuno-PET using anticarcinoembryonic antigen bispecific antibody and ${ }^{68} \mathrm{Ga}$-labeled peptide in metastatic medullary thyroid carcinoma: clinical optimization of the pretargeting parameters in a first-in-human trial. J Nucl Med. 2016;57:1505-1511. 